\title{
ATENÇÃO MULTIPROFISSIONAL AO IDOSO EM CONDIÇÃO CRÔNICA NA ESTRATÉGIA SAÚDE DA FAMÍLIA
}

\author{
Multiprofessional care for the elderly in a chronic condition in the Family Health \\ Strategy
}

\section{Atención multiprofesional al mayor de la Estrategia Salud de la Familia con condición crónica}

\author{
Andressa Christiny da Silva Ferreira (Lattes) \\ Universidade Federal de Mato Grosso - UFMT - Rondonópolis (MT) - Brasil \\ Magda de Mattos (Lattes) \\ Universidade Federal de Mato Grosso - UFMT - Rondonópolis (MT) - Brasil
}

\section{RESUMO}

Objetivo: Identificar a percepção da equipe multiprofissional sobre atenção à saúde dos idosos em condição crônica. Métodos: Trata-se de uma pesquisa de abordagem qualitativa, desenvolvida em duas Unidades de Saúde da Família localizadas em um município da região sul do estado de Mato Grosso, Brasil, com nove profissionais de saúde. A coleta de dados ocorreu no período de fevereiro a julho de 2017, por meio de entrevista semiestruturada. De acordo com a análise de conteúdo, emergiram duas categorias: "Conhecimento teórico sobre o idoso crônico" e "Vivenciando a prática na promoção e prevenção de agravos à saúde dos idosos". Resultados: Os profissionais demonstraram conhecer os principais aspectos que envolvem a condição crônica do idoso, contudo a atenção multiprofissional à saúde do idoso na Estratégia de Saúde da Família é uma prática fragmentada no cuidado e na educação em saúde. Conclusão: A atenção multiprofissional ao idoso em condição crônica ainda necessita de um envolvimento paritário de todos os seus membros, e carece de um maior conhecimento teórico e envolvimento da equipe com observação sistemática da política de promoção à saúde do idoso, a fim de realizar um atendimento de qualidade a esse grupo etário.

Descritores: Idoso; Doença Crônica; Assistência à Saúde; Estratégia Saúde da Família.

\begin{abstract}
Objective: To identify the multiprofessional team's perception of health care for the elderly in a chronic condition. Methods: This is a qualitative research, developed in two Family Health Units, in a municipality in the southern region of the state of Mato Grosso, Cuiabá, Brazil, with nine health professionals. Data collection was carried out in the period from February to July 2017, by means of semi-structured interviews. According to content analysis, two categories have emerged: "Theoretical knowledge about the elderly with chronic conditions" and "Experiencing the practice in the promotion and prevention of health concerns affecting seniors". Results: The professionals demonstrated understanding the main aspects that involve the chronic condition of the elderly; however, the multiprofessional health care for the elderly in the Family Health Strategy is a fragmented practice in health care and health education. Conclusion: The multiprofessional care for the elderly in chronic condition still requires a parity-based involvement of all its members, and lacks greater theoretical knowledge and greater involvement of the team with systematic observation of the health promotion policy for the elderly, in order to provide quality care to this age group.
\end{abstract}

Descriptors: Aged; Chronic Disease; Health Care; Family Health Strategy. 


\section{RESUMEN}

Objetivo: Identificar la percepción del equipo multiprofesional sobre la atención a la salud de los mayores con condición crónica. Métodos: Se trata de una investigación de abordaje cualitativo desarrollada en dos Unidades de Salud de la Familia localizadas en un municipio de la región sur del estado de Mato Grosso, Brasil, con nueve profesionales sanitarios. La recogida de datos se dio en el periodo entre febrero y julio de 2017 a través de entrevista semi-estructurada. Emergieron dos categorías según el análisis de contenido: "Conocimiento teórico sobre el mayor crónico" y "Vivenciando la práctica para la promoción y prevención de agravios a la salud de los mayores". Resultados: Los profesionales han demostrado conocer los principales aspectos de la condición crónica del mayor, sin embargo, la atención multiprofesional a la salud del mayor en la Estrategia Salud de la familia es una práctica fragmentada en el cuidado y la educación en salud. Conclusión: La atención multiprofesional al mayor con condición crónica aún necesita del envolvimiento paritario de todos sus miembros y hace falta más conocimiento teórico y el envolvimiento del equipo con observación sistemática de la política de promoción para la salud del mayor para una atención de calidad con ese grupo etario.

Descriptores: Anciano; Enfermedad Crónica; Prestación de Atención de Salud; Estrategia Salud Familiar.

\section{INTRODUÇÃO}

O envelhecimento populacional é uma realidade mundial e decorre, dentre outros fatores, da redução das taxas de mortalidade e natalidade, e do aumento da expectativa de vida ${ }^{(1)}$. As estimativas da Organização Mundial de Saúde (OMS) apontam que o Brasil terá mais de 64 milhões de idosos até o ano de 2050(2). Em vez de ser tratado como um problema, o aumento da longevidade deve ser encarado como uma conquista da humanidade ${ }^{(3)}$.

Nesse sentido, os dados demográficos despontam a necessidade urgente dos gestores observarem essa transição e, juntamente com a sociedade, debaterem a Política Pública de Promoção da Saúde ${ }^{(4)}$, criada pela Portaria GM/ $M S n^{\circ} 687$, de 30 de março de 2006, no intuito de avaliar o que está proposto para essa população, integrada com a Política Nacional de Saúde da Pessoa Idosa, criada especificamente para desenvolver ações de promoção da saúde e prevenção de agravos à pessoa idosa, aprovada pela Portaria $n^{\circ} .2 .528$, de 19 de outubro de 2006, que considera um indivíduo idoso aquele com idade igual ou superior a 60 anos e tem por finalidade a recuperação, manutenção e promoção da autonomia e a independência dos idosos, direcionando medidas coletivas e individuais de saúde para esse fim, em consonância com os princípios e diretrizes do Sistema Único de Saúde (SUS) ${ }^{(5)}$.

O envelhecimento é um processo natural, que compreende uma etapa da vida e se configura por mudanças físicas, psicológicas e sociais. A esse respeito, considera-se que o envelhecer pode provocar limitações da capacidade funcional, cognitiva, sensorial e afastamento do convívio social ${ }^{(5,6)}$. É a fase em que a pessoa se depara, geralmente, com os objetivos de vida alcançados, mas também com muitas perdas, destacando-se a condição de saúde como um dos aspectos que pode ser afetado(6).

Com a longevidade dos idosos, situações de adoecimento também podem acompanhar esse processo, como as condições crônicas, que possuem características de início e evolução lentos, e apresentam como principais fatores de risco o sedentarismo, a alimentação inadequada, a ingestão de bebidas alcóolicas, a hereditariedade e a exposição a fatores ambientais ou envelhecimento ${ }^{(5-7)}$.

Caracterizam-se como condição crônica as doenças crônicas não transmissíveis (DCNT), as doenças infecciosas persistentes, os distúrbios mentais, a deficiência física e as condições ligadas à manutenção da saúde por ciclos de vida( ${ }^{(7)}$, representando um alto ônus, tanto para os indivíduos e familiares quanto para o sistema de saúde, em virtude dos custos com cuidados e sobrecarga medicamentosa ${ }^{(6)}$. Para tanto, entende-se a necessidade de ações contínuas, proativas e integradas, do sistema de atenção à saúde, dos profissionais e das pessoas usuárias para o seu controle efetivo, eficiente e com qualidade ${ }^{(7,8)}$.

Nessa perspectiva, a assistência aos idosos em condição crônica envolve uma equipe multiprofissional, cujo atendimento deve ser fundamentado em diretrizes clínicas, baseado em evidências de informações relevantes e ações organizadas para que estes recebam a atenção adequada. Essas ações podem ser individuais ou em grupos, incluem a prevenção de doenças e agravos, e o estímulo ao autocuidado ${ }^{(7-9)}$.

A Atenção Primária à Saúde (APS) configura-se como porta de entrada para o SUS, em que aproximadamente $80 \%$ das necessidades de atenção à saúde de uma comunidade adstrita devem ser abordadas de maneira resolutiva. Sendo assim, é na Estratégia de Saúde da Família (ESF) que o caráter multiprofissional tem sido determinante para 
a ampliação da visão acerca do processo saúde-doença do idoso em condição crônica. A partir disso, o atendimento a esses indivíduos se tornou cada vez mais preponderante, em face da necessidade de um acompanhamento contínuo para melhorar as condições de saúde e qualidade de vida(7,10).

A atenção à saúde do idoso em condição crônica, na ESF, deve pautar-se em um modelo de cuidado com foco nos direitos, necessidades, preferências e habilidades desses usuários, por meio de uma assistência multiprofissional humanizada, criando vínculos pautados na ética, compromisso e respeito. Contudo, ainda há necessidade de mecanismos de sensibilização e ação social quantos aos aspectos relacionados à prevenção e à promoção de saúde para um envelhecer saudável ${ }^{(5,8,11)}$. Assim, dentro da equipe multiprofissional, é necessário que todos os membros estejam comprometidos com a qualidade do trabalho, com uma boa comunicação com a equipe, com um ambiente organizado e com ações planejadas, desde o primeiro contato até o final do atendimento(5).

Diante de uma perspectiva de atenção multiprofissional aos idosos nas ESF, este estudo tem como questionamento: "Qual a percepção da equipe multiprofissional sobre o atendimento aos idosos em condição crônica nas unidades da Estratégia de Saúde da Família?". Nesse contexto, o objetivo do presente estudo é identificar a percepção da equipe multiprofissional sobre a atenção à saúde dos idosos em condição crônica.

\section{MÉTODOS}

Trata-se de uma pesquisa de campo, de abordagem qualitativa ${ }^{(12)}$, pois, de acordo com o objetivo proposto, permite aos pesquisadores compreenderem os fatos e fenômenos que envolvem a atenção multiprofissional ao idoso em condição crônica. Este estudo justifica-se pela observação das autoras, no cotidiano de trabalho dos profissionais na ESF, no atendimento de idosos em condição crônica, mesmo com a política de atenção integral a essa população para a promoção de um envelhecimento saudável e a prevenção de agravos à saúde, que a assistência ainda ocorre de forma fragmentada e por meio de ações pontuais.

Este estudo ocorreu em duas unidades da ESF do município de Rondonópolis, Mato Grosso, Brasil, localizado na região sudeste do estado. Os participantes da pesquisa foram nove profissionais de saúde, selecionados de forma intencional(13), distribuídos da seguinte forma: dois médicos, duas enfermeiras, um cirurgião-dentista, dois técnicos de enfermagem e dois agentes comunitários de saúde, cujas ações estavam relacionadas à atenção multiprofissional ao idoso em condição crônica. Como critérios para participação no estudo, deveriam ser: profissionais da equipe multiprofissional que atuassem diretamente com os idosos. Não fizeram parte os profissionais que não aceitaram colaborar com a pesquisa e/ou que estivessem ausentes das ESF por motivo de licença ou férias no período da coleta de dados.

O município em questão está localizado a 210 km da capital Cuiabá e possui uma população estimada em 222.316 habitantes ${ }^{(14)}$. Atualmente, dispõe de $39 \mathrm{ESF}^{(15)}$ divididas em cinco distritos, conforme sua localização geográfica, sendo distritos norte, leste, centro-oeste, oeste e sul. Os bairros onde se localizam as unidades selecionadas têm, entre suas características, uma população com baixa condição socioeconômica.

Estão cadastrados 807 idosos nas duas unidades selecionadas, dos quais 475 possuem diagnóstico médico de diabetes mellitus (DM) e/ou hipertensão arterial sistêmica (HAS), de acordo com os dados dos prontuários dos pacientes, cujo atendimento ocorre por meio de agendamento e de acordo com as especificidades e rotina de cada serviço. As equipes de saúde que compõem as ESF possuem os seguintes profissionais atuantes: médico, enfermeiro, técnicos de enfermagem e agentes comunitários de saúde (ACS), e em apenas uma das unidades há cirurgião-dentista.

A coleta de dados ocorreu no período de fevereiro a julho de 2017, por meio de entrevista semiestruturada, sendo gravada e transcrita na íntegra. A entrevista teve duas etapas. A primeira compôs as variáveis: categoria profissional, idade, sexo, tempo de atuação na ESF. A segunda etapa continha as seguintes questões norteadoras: Qual seu conhecimento teórico sobre um idoso em condição crônica? Quais as atividades desenvolvidas pela equipe na prática para promoção e prevenção de agravos à saúde desses idosos?

As entrevistas foram efetuadas na própria ESF, de acordo com agendamento prévio e disponibilidade do profissional, sendo realizada em local privado e com duração de 30 minutos cada uma.

Para a análise dos dados acerca da atenção à saúde ao idoso pela equipe multiprofissional, após a coleta do material de pesquisa, sistematizou-se e submeteu-se a uma pré-análise para reorganização das ideias iniciais, de forma a possibilitar a análise e consequente interpretação, conforme o método de análise de conteúdo ${ }^{(16)}$. Emergiram as categorias: "Conhecimento teórico sobre o idoso crônico" e "Vivenciando a prática na promoção e prevenção de agravos à saúde dos idosos". 
Respeitaram-se os aspectos éticos durante a pesquisa, de acordo com a Resolução 466/2012, iniciando-se somente após a aprovação do Comitê de Ética em Pesquisa da Universidade Federal de Mato Grosso, Parecer $\mathrm{n}^{\circ}$. 1.931.881. Todos assinaram o Termo de Consentimento Livre e Esclarecido e, para manter o anonimato, os profissionais foram denominados pelas letras iniciais da profissão, sendo: agente comunitário de saúde (ACS), enfermeiro (Enf.), médico (Med.), cirurgião-dentista (Odo.) e técnico de enfermagem (TE), enumerados sequencialmente de acordo com a ordem da entrevista.

\section{RESULTADOS E DISCUSSÃO}

A seguir, serão apresentados os dados de caracterização dos participantes do estudo e, em seguida, as categorias que emergiram a partir da análise do material coletado. Na categoria "Conhecimento teórico sobre o idoso crônico", agruparam-se os fragmentos de falas acerca dos conhecimentos da equipe sobre condição crônica. Na categoria "Vivenciando a prática na promoção e prevenção de agravos à saúde dos idosos", a ênfase dada foi nas atividades desenvolvidas pela equipe para promoção e prevenção de agravos à saúde dos idosos.

\section{Caracterização dos participantes do estudo}

O grupo de nove participantes do estudo foi composto por duas enfermeiras, duas agentes comunitárias de saúde, duas médicas, duas técnicas de enfermagem e um cirurgião-dentista. A maioria do grupo era do sexo feminino, com idade entre 25 e 43 anos.

No que concerne ao tempo de atuação profissional na ESF, observou-se que os profissionais com maior tempo são os técnicos de enfermagem e ACS, os demais possuem período de atuação variando de meses a poucos anos, o que permite deduzir que existe maior rotatividade entre os profissionais enfermeiros e médicos.

A ESF, enquanto proposta de reordenação das práticas de saúde nas comunidades, tem alcançado resultados expressivos em muitos municípios, contudo tem apresentado problemas relevantes, entre os quais a rotatividade de profissionais, como observado no presente estudo. Entende-se que a alternância de membros da equipe de saúde constitui-se um grande desafio para os gestores, tendo em vista a dificuldade em manter a mesma equipe por um período prolongado, tornando-se altamente prejudicial para a efetivação das ações de saúde, como a criação de vínculo com a população( ${ }^{(17)}$.

\section{Conhecimento teórico sobre o idoso crônico}

Nesta categoria, emergiu o conhecimento teórico da equipe multiprofissional acerca da condição crônica do idoso. Observou-se, assim, que aspectos relacionados à evolução, etiologia e capacidade funcional foram elencados.

Para que a equipe multiprofissional atue na promoção da saúde e na prevenção de doenças e agravos na atenção aos idosos, é indispensável que os profissionais compreendam a importância da sua função e busquem continuamente por atualização dos conhecimentos, o que lhes permitirá prestar uma assistência integral e equânime à população adstrita(7).

Dessa forma, neste estudo, buscou-se saber qual era a compreensão da equipe multiprofissional acerca da condição crônica do idoso:

"Acho que, na condição crônica, os idosos dependem totalmente de outra pessoa." (TE1)

"São doenças que a pessoa tem para o resto da vida, tem que cuidar mais." (TE2)

"Uma patologia ou doença que o indivíduo tem por muito tempo." (Odo)

"A pessoa que possui uma condição crônica necessita de cuidado contínuo e acompanhamento. Inclusive, a equipe de saúde é muito importante pra esse paciente em condição crônica." (Med.2)

"Condição crônica é uma situação que o paciente se debilita por fatores genéticos ou externos. Paciente que requer um cuidado contínuo." (Med.1)

Para alguns dos profissionais de saúde, participantes do atual estudo, a definição de condição crônica envolve características como temporalidade, necessidade de cuidados contínuos e a dependência de outros. A condição crônica pode ser considerada como experiência de vida que envolve permanência e desvio do normal, causados por patologias que acarretam perdas e disfunções, além de permanente alteração no cotidiano das pessoas. Expressa, ainda, que essa permanência causa estresse devido à alteração da imagem corporal, necessidade de adequação 
social e psicológica, além de mudança na expectativa de vida. Tem como principais características a necessidade de estabilização da condição e o autocuidado apoiado ${ }^{(7,9)}$.

As diversas alterações que acometem os indivíduos decorrentes das condições crônicas devem ser de conhecimento de todos os profissionais, haja vista que o planejamento e execução de atividades de promoção à saúde e prevenção de agravos implicam em saber como atuar diante da complexidade que abarca o cuidado à pessoa idosa. A esse respeito, estudos têm elencado diferentes conhecimentos necessários à equipe, como o processo de envelhecimento, o adoecimento crônico, tecnologias de cuidado e o papel dos cuidadores e/ou familiares ${ }^{(7,18,19)}$.

Observa-se que, no Brasil, juntamente com o prolongamento da vida, vêm acontecendo importantes modificações no nível de saúde da população, advertindo que o envelhecimento ocorre a partir da interação de um acúmulo de processos sociais, biológicos e comportamentais ao longo da vida, e que, por isso, a situação de saúde e doença da população necessita de avaliações rigorosas e ações interdisciplinares. Por esse motivo, a preocupação com as doenças infectocontagiosas perde lugar para a alta prevalência das condições crônicas ${ }^{(6,8)}$. Visto isso, os profissionais da presente pesquisa destacam em suas falas que um idoso em condição crônica demanda para seus cuidados uma atenção maior, que não envolve apenas a equipe de saúde, mas também o cuidador e o próprio idoso em seu tratamento:

"O idoso tem que ter uma atenção maior de todos da família, de todo mundo que está por perto." (ACS1)

"[...] Aquele paciente que requer um cuidado contínuo, tem que ser mais acompanhado. Os cuidadores devem estar mais envolvidos com o tratamento." (Med.1)

Nos fragmentos de falas anteriores, observa-se que, para os profissionais, a condição crônica, quando associada a pessoas idosas, demanda uma atenção que não envolve apenas o tratamento medicamentoso e a equipe de saúde da ESF, mas também família, tempo, relações humanas e cuidados contínuos.

Para o idoso em condição crônica, é necessária uma atenção integral que envolve os cenários de saúde e profissionais, além de orientações para o autocuidado. Nessa perspectiva, os indivíduos e suas famílias necessitam de apoio em suas comunidades e de políticas abrangentes e sistemáticas para a prevenção ou gerenciamento eficaz do estado de saúde do indivíduo, bem como no manejo dos sintomas, tratamento, consequências físicas e psicológicas, e mudanças no estilo de vida(8).

No presente estudo, a equipe ressalta a importância do envolvimento dos cuidadores na assistência à pessoa idosa em condição crônica. O impacto de vivenciar o cuidado a essas pessoas no núcleo da família tem sido objeto de estudo(20), com destaque para que a equipe multiprofissional conheça e identifique os sentimentos e necessidades, principalmente quando ocorre uma nova configuração da estrutura familiar, por exemplo, diante do agravamento da doença e internação do paciente.

De acordo com a Organização Mundial de Saúde (OMS), existem algumas estratégias que possibilitam melhorar a atenção multiprofissional aos idosos em condição crônica, como a expansão de informações e conhecimento entre profissionais/usuários e entre profissionais/cuidadores/família, além de orientações educativas para melhorar o manejo dessa condição, como a adesão ao tratamento e o autocuidado(8).

No entanto, para que essas estratégias sejam efetivas, é necessário o conhecimento cientifico por parte dos profissionais quanto ao conceito de condição crônica, tratamento e acompanhamento adequado desse público, pois, sem este, a prática não será pautada em um saber cientifico, nem nas reais necessidades da população idosa(6). Os profissionais necessitam, também, conhecer quais são as DCNT, pois não se resumem somente ao DM e HAS. Necessitam, portanto, ter habilidade para o cuidado com os idosos portadores de outras condições crônicas.

\section{Vivenciando a prática na promoção e prevenção de agravos à saúde dos idosos}

Esta categoria descreve como os profissionais da equipe da ESF vislumbram os cuidados prestados aos idosos, visando à promoção e prevenção de agravos à saúde. A necessidade de uma atenção contínua à saúde dessa população requer diferentes níveis de intervenção dos serviços de saúde, permeados por uma equipe multiprofissional, adequados às distintas fases da enfermidade e ao grau de incapacidade ${ }^{(9)}$.

Assim, ao vivenciarem no cotidiano de trabalho da assistência aos idosos em condição crônica, o atendimento dessa população foi mencionado por meio de ações desenvolvidas por parte da equipe, como o grupo de caminhada, no qual nem todos os profissionais se envolvem, como pode ser visto a seguir, pelas falas:

"A gente tem o grupo de caminhada toda terça-feira e sexta-feira de manhã. Antes de caminhar, as ACS fazem um alongamento e uma oração com os idosos e caminhamos, só não vamos muito longe, sempre vai com a 
gente uma técnica de enfermagem ou a enfermeira." (ACS2)

"Tem a caminhada para os idosos. E acredito que deve focar mais nele, pois é um grupo muito importante para esses idosos. Por enquanto, tem só duas vezes na semana. Da caminhada, os idosos gostam muito." (TE2)

Atividades físicas, como a caminhada, são importantes mecanismos de prevenção e promoção à saúde dos idosos, contudo impõe aos profissionais uma capacidade técnica e conhecimentos teóricos para realizar tais atividades ${ }^{(21)}$. Sendo assim, a atividade física, quando realizada de forma regular, contribui para melhorar a qualidade de vida do idoso, proporcionando um condicionamento físico e tornando-o capaz de exercer seus afazeres cotidianos ao longo da vida ${ }^{(7,22,23)}$.

Estimular a população idosa à adesão de uma atividade física também contribui para a prevenção/controle de doenças cardiovasculares, pois a inatividade física e/ou falta de exercícios regulares é um dos fatores de risco comportamentais para o desenvolvimento da hipertensão arterial( ${ }^{(24)} \mathrm{e}$ de outros problemas de saúde relacionados às condições crônicas $^{(19)}$. Nesse sentido, estimular a prática de exercício físico regular é essencial, pois seus benefícios são inquestionáveis na promoção da saúde e qualidade de vida. Ademais, entende-se que é primordial estimular os idosos que já possuem o hábito de se exercitar para que continuem ${ }^{(20)}$.

Muito embora tenha sido levantada a questão da caminhada, ela ainda é uma atividade pontual nessas unidades, como se observa a seguir:

"Só tem a caminhada. Não tem mais nada e não são muitos idosos que participam. Temos uma academia popular, mas ela não é coberta, não tem nem como os idosos ficarem lá e também não temos profissionais para auxiliar esses idosos." (TE2)

A fala anterior demonstra uma realidade em que as condições de infraestrutura são precárias e há ausência de outros profissionais, como um educador físico. É importante destacar que o local em que o estudo foi realizado localiza-se em uma região de condição climática muito quente, o que dificulta atividades ao ar livre.

A efetivação de qualquer atividade na ESF perpassa pelo planejamento de ações com a participação de toda a equipe. Contudo, o que tem se observado é o pouco envolvimento de alguns profissionais da saúde ${ }^{(22)}$, gerando, consequentemente, certo desestímulo aos demais membros. Não obstante, a prática de cuidados às pessoas idosas exige "abordagem global, interdisciplinar e multidimensional", com destaque para a interação entre os aspectos físicos, psíquicos e sociais que permeiam a saúde dos idosos ${ }^{(5)}$.

Outro aspecto relacionado ao atendimento do idoso refere-se às consultas médicas, geralmente previamente agendadas, principalmente para aqueles pacientes portadores de doenças crônicas, como diabetes e hipertensão:

"Tem o grupo que eles [idosos] podem vir para agendar o atendimento, toda quinta-feira." (ACS2)

"Em relação à organização [do atendimento ao idoso], tem o dia do hipertenso e/ou diabético, que é na terça. E na quinta que é saúde do idoso, onde alguns que têm hipertensão e/ou diabetes também vêm." (Enf.2)

"Eu acho que é bem acompanhado, medicado, acompanhado pelo médico. A gente consegue acompanhar ele e acompanhar as medicações." (ACS1)

Nessas falas, observa-se que ainda há profissionais que possuem uma visão focada no modelo curativista. A organização da assistência ao idoso em condição crônica de forma fragmentada, que não supre a necessidade de ser acompanhado por uma equipe multiprofissional. De acordo com a Política Nacional de Saúde da Pessoa Idosa, o modelo de atenção baseado na assistência médica individual não é eficaz para a promoção e prevenção aos agravos à saúde dos idosos ${ }^{(5)}$. As condições crônicas requerem um esforço contínuo, sustentável e em longo prazo, com estratégias abrangentes e duradouras, que afetem e reflitam a estrutura, os serviços, os sistemas de saúde e as necessidades reais da população(9).

Na prática da equipe de saúde na atenção primária, o foco de atenção ainda está centrado em abordagens individuais, com algumas práticas que envolvem a prevenção ${ }^{(25)}$. Para tanto, entende-se a necessidade de aprofundar estudos que abordem propostas de melhoria das condições de saúde da população como um todo, e não de forma individualizada, principalmente para as condições crônicas, como câncer, demência e outras doenças oriundas do envelhecimento(25).

Vê-se, então, que o modelo de atenção à saúde do idoso nas unidades da atual pesquisa é, predominantemente, caracterizado pela fragmentação do cuidado, com centralização do poder no profissional médico. 
Outro aspecto, também relevante, que surgiu no espaço de trabalho na ESF é que, para alguns profissionais, a criação de vínculo entre profissional/idoso e profissional/família é fundamental na assistência ao idoso em condição crônica:

"[...] É muito importante a gente ter um laço muito bom, tanto com o idoso quanto com a família, para descobrir mais detalhes sobre a vida dele." (Odo.)

"[...] Você tem que ter carinho no que faz, tem que ter amor, tem que pegar na mão, olhar nos olhos, tem que abraçar. Idoso gosta muito disso e isso é muito importante, não só porque é idoso e você tem que fazer algo, mas eu acho que o médico tem que ter esse contato, e isso gera confiança [...]." (Med.2)

"A família também é a base para a melhora, a reabilitação do paciente, porque, se o paciente, às vezes, for dependente e não tiver o apoio da família, ou nem precisa ter uma dependência, mas se a família não auxiliar de certa forma, o tratamento não vai ter o esperado não, não vai ser adequado com o que a gente espera." (Enf.1)

O processo de acolhimento é um ato que implica em bem receber e escutar, oferecer proteção, amparo, e dar resposta capaz de resolver o problema apresentado pelo indivíduo. Representa a interação entre o profissional de saúde/usuário/família e se conforma como uma prática permeada de ações comunicacionais. Portanto, a equipe multiprofissional na ESF tem por atribuição o estabelecimento de um vínculo com a população, possibilitando o compromisso e a continuidade da atenção, o que configura um novo modo de agir, em que as responsabilidades pela atenção à saúde devem ser compartilhadas entre as famílias e as equipes ${ }^{(17,26,27)}$.

A família, além de vivenciar os conflitos naturais do próprio ciclo vital de seus familiares, também se depara com diferentes adversidades, como a condição crônica de um familiar, que carece de olhar próximo e responsável dos profissionais de saúde. Nesses casos, o cuidado necessita ser frequente e sistemático, objetivando a melhoria da qualidade de vida da família como um todo, respeitando os direitos humanos e sociais ${ }^{(28)}$.

A conduta de alguns profissionais no acolhimento aos idosos na ESF é identificada, especificamente por um dos membros da equipe multiprofissional, como inadequada:

"Nem todos acolhem o idoso como deve ser. Muitos não têm paciência, nem escutam, viram as costas e os deixam falando sozinhos." (TE2)

Quando não há acolhimento baseado na escuta qualificada, capaz de atender às necessidades do usuário, dificulta-se a garantia de acesso oportuno às tecnologias adequadas. Isto impede, por exemplo, que todos sejam atendidos com prioridade a partir da avaliação de vulnerabilidade, gravidade e risco ${ }^{(29,30)}$. Portanto, a consolidação do acolhimento e o vínculo entre profissionais/idosos/familiares é de fundamental importância para que as ações da equipe multiprofissional alcancem o intuito. Nesse caso, o de prestar uma atenção integral e equânime aos idosos em condição crônica.

Também se destacou entre a equipe multiprofissional a visita domiciliária como uma atividade pertinente ao processo de atenção à saúde do idoso em condição crônica:

"[...] Tem a visita domiciliar. Se necessário também, a gente faz esse tipo de atendimento." (Enf.1)

"[...] Têm as visitas dos ACS com o enfermeiro e médico juntos, então a gente consegue envolver a família, que também colabora e ajuda." (ACS1)

"[...] Têm as visitas que, geralmente, eu faço mais para idosos em condição crônica, porque tem incapacidade de vir até a unidade." (Med.2)

Dentre as atividades de responsabilidade da equipe multiprofissional na Política Nacional de Atenção Básica (PNAB), a visita domiciliária contribui para ações de promoção, prevenção das doenças e agravos, e de vigilância à saúde ${ }^{(31)}$. Fato esse que, provavelmente, contribuiu para que essa atividade tenha sido mencionada pelos participantes do presente estudo.

A visita domiciliária é uma ferramenta muito importante de atenção à saúde ao idoso em condição crônica na ESF, pois permite o desenvolvimento e o fortalecimento do vínculo e a prevenção de possíveis complicações decorrentes do processo de adoecimento ${ }^{(10)}$. Esse instrumento possibilita aos profissionais de saúde a realização de um acompanhamento sistemático do idoso e de seus familiares, bem como a elaboração de um plano de cuidado individualizado(32).

Para tanto, os profissionais que atuam na ESF devem ter clareza quanto à importância da manutenção do idoso na rotina familiar e na vida em comunidade, de forma participativa e construtiva ${ }^{(25)}$, principalmente aquele em 
condição crônica, que demanda uma assistência multiprofissional. Assim, os resultados da pesquisa em questão permitem sugerir que os idosos em condição crônica ainda carecem de ações da equipe multiprofissional, pois os conhecimentos e atividades apresentadas pelos participantes dessa pesquisa não contemplam em sua totalidade as diretrizes da Política Nacional de Saúde da Pessoa Idosa. Dessa forma, os resultados evidenciam que as condições relacionadas ao atendimento ao idoso são passiveis de intervenção, o que é fundamental para a prevenção e promoção da saúde deles, evitando desfechos clínicos adversos, especialmente no que diz respeito às doenças crônicas. O conhecimento dos profissionais sobre os fatores associados a essas doenças em idosos permite que ações de saúde propostas a esse grupo possam ser desenvolvidas.

Assim, algumas iniciativas integradas com a intersetorialidade, com os demais níveis de complexidade, com as escolas, as universidades, a comunidade e outros setores, são importantes para discutir as ações de intervenção no atendimento ao idoso. Sugere-se, inicialmente, buscar identificar as vulnerabilidades desse grupo populacional, conhecer as condições sociais, econômicas e como se dá o acesso a ESF. Além disso, planejar a capacitação da equipe multiprofissional para atenção ao cuidado ao idoso; integrar um educador físico à equipe multiprofissional; possibilitar um conhecimento mais aprofundado da equipe sobre a Política Nacional de Promoção da Saúde ${ }^{(4)}$ e sua articulação com a Política Nacional de Promoção da Saúde do Idoso(5); utilizar de forma efetiva a Caderneta de Saúde da Pessoa Idosa ${ }^{(33)}$; usar sistematicamente o Caderno de Atenção Básica sobre Envelhecimento da Pessoa Idosa ${ }^{(34)}$, e incentivar a equipe para a visita domiciliar ao idoso em condição crônica. A caderneta permite identificar situações de risco para a saúde das pessoas idosas, informações sobre a sua condição de saúde, o suporte familiar e social, fornecendo subsídios técnicos que ajudarão na qualificação da prática diária das equipes de saúde, em especial dos profissionais da atenção básica(21).

Nesse delineamento, ressalta-se também a criação de grupos de Sala de Espera, pois podem conceber um local excelente de discussão entre profissionais, idosos e familiares, proporcionando educação em saúde por meio das vivências e trocas de experiência. Esse processo de educação em saúde ampara os familiares e o idoso para a responsabilidade do autocuidado, a promoção em saúde e o enfrentamento do familiar de ser cuidador de uma pessoa com condição crônica.

Assim, espera-se que as ações propostas no atual estudo para os profissionais de saúde convenham para reorientar os serviços de saúde, sendo o início de um trabalho para tentar, inicialmente, minorar a fragmentação da assistência ao idoso baseada com foco na doença, indo em direção à perspectiva da atenção integral ao idoso.

A despeito das intervenções propostas anteriormente, como resultados, esperam-se impactos positivos na melhora das condições de saúde dos idosos crônicos, bem como a implementação de atividades de forma sistemática na busca do envelhecimento saudável da população adstrita. Para tanto, entende-se a necessidade de avaliações permanentes a despeito das atividades desenvolvidas pelos profissionais, bem como de avaliações dos indicadores do envelhecimento, como a capacidade funcional (física, mental e social), a autonomia, a ausência ou baixo índice de agravos à saúde, dentre outros.

Entre as limitações do presente estudo, destaca-se o fato de ter sido realizado somente em duas ESF, contudo, estende-se a sua importância, visto o número significativo de idosos em condição crônica que dependem de uma assistência multiprofissional equânime e resolutiva.

\section{CONSIDERAÇÕES FINAIS}

Por meio do estudo foi possível observar que os participantes entendem a condição crônica quanto à temporalidade, necessidade de cuidado contínuo e a importância do envolvimento da família/cuidador nesse processo. Contudo, destaca-se que a atenção multiprofissional ao idoso em condição crônica nas duas Estratégia Saúde da Família investigadas ainda necessita de um envolvimento igualitário de todos os membros, com foco na prevenção e promoção da saúde dessa população.

A partir dos achados do estudo, sugerem-se ações com foco nos profissionais, para que reconheçam e assumam a responsabilidade quanto aos cuidados aos idosos crônicos, bem como para a promoção de um envelhecimento saudável da população como um todo.

\section{CONFLITOS DE INTERESSE}

Os autores informam que não há conflitos de interesse no estudo realizado. 


\section{CONTRIBUIÇÕES:}

Andressa Christiny da Silva Ferreira participou do desenho do estudo, da coleta, organização, análise e discussão dos dados, e da redação do artigo. Magda de Mattos participou do desenho do estudo, da análise e discussão dos dados, da redação do artigo e das revisões finais.

\section{REFERÊNCIAS}

1. Miranda GMD, Mendes ACG, Silva ALA. O envelhecimento populacional brasileiro: desafios e consequências sociais atuais e futuras. Rev Bras Geriatr Gerontol. 2016;19(3):507-19.

2. Organização Mundial de Saúde. Compartilhando responsabilidades na promoção de justiça, Sinus, 2014. Brasília: Ministério da Saúde; 2015.

3. Silva MRF. Envelhecimento e proteção social: aproximações entre Brasil, América Latina e Portugal. Serv Soc Soc. 2016;(126):215-34.

4. Brasil. Ministério da Saúde. Portaria GM/MS n 687, de 30 de março de 2006. Institui a Política Nacional de Promoção da Saúde [acesso em 2018 Jun 20]. Disponível em: http://bvsms.saude.gov.br/bvs/saudelegis/gm/2006/ prt0687_30_03_2006.html

5. Brasil. Ministério da Saúde. Portaria $n^{0} 2.528$ de 19 de outubro de 2006. Aprova a Política Nacional de Saúde da Pessoa Idosa [acesso em 2018 Jun 20]. Disponível em: http://bvsms.saude.gov.br/bvs/saudelegis/gm/2006/ prt2528_19_10_2006.html

6. Valcarenghi RV, Lourenço LFL, Siewertl JS, Alvarez AM. Produção científica da Enfermagem sobre promoção de saúde, condição crônica e envelhecimento. Rev Bras Enferm. 2015;68(4):705-12.

7. Mendes EV. O cuidado das condições crônicas na atenção primária à saúde: o imperativo da consolidação da estratégia da saúde da família. O cuidado das condições crônicas na atenção primária à saúde: o imperativo da consolidação da estratégia da saúde da família. Brasília: Organização Pan-Americana da Saúde; 2012.

8. Silva CG, Sena LB, Rolim ILTP, Sousa SMA, Sardinha AHL. Cuidados de enfermagem a pacientes com condições crônicas de saúde: uma revisão integrativa. Rev Pesqui Cuid Fundam. 2017;9(2):599-605.

9. Goulart FAA. Doenças crônicas não transmissíveis: estratégias de controle e desafios e para os sistemas de saúde. Brasília: Ministério da Saúde; 2012.

10. Duncan BB, Chor D, Aquino EML, Bensenor IM, Mill JG, Schmidt MI, et al. Doenças Crônicas Não Transmissíveis no Brasil: prioridade para enfrentamento e investigação. Rev Saúde Pública. 2012; 46(Supl 1):126-34.

11. Vello LS, Popim RC, Carazzai EM, Pereira MAO. Saúde do idoso: percepções relacionadas ao atendimento. Escola Anna Nery Revista de Enfermagem. 2014;18(2):330-5.

12. Minayo MCS. O desafio do conhecimento: pesquisa qualitativa em saúde. 14ª ed. São Paulo: Hucitec; 2014.

13. Bussab WO, Morettin PA. Estatística básica. São Paulo: Saraiva; 2002.

14. Instituto Brasileiro de Geografia e Estatística. Estimativas da população dos municípios brasileiros com data de referencia em $1^{\circ}$ de julho de 2017 [Internet]. Rio de Janeiro; 2017 [acesso em 2018 Jun 20]. Disponível em: https://cidades.ibge.gov.br/xtras/perfil.php?lang=\&codmun=510760

15. Ministério da Saúde (BR). CNES: Cadastro Nacional de Estabelecimentos de Saúde [acesso em 2018 Jun 20]. Disponível em: http://datasus.saude.gov.br/sistemas-e-aplicativos/cadastros-nacionais/cnes

16. Bardin L. Análise de conteúdo. Lisboa: Edições 70; 2011.

17. Carnut L. Cuidado, integralidade e atenção primária: articulação essencial para refletir sobre o setor saúde no Brasil. Saúde Debate. 2017;41(115):1177-86.

18. Leite MT, Pai SD, Quintana JM, Costa MC. Doenças crônicas não transmissíveis em idosos: saberes e ações de agentes comunitários de saúde. Rev Pesqui Cuid Fundam. 2015;7(2):2263-76.

19. World Health Organization. World report on ageing and health. [acesso em 2018 Jul 02]. Disponível em: http:// apps.who.int/iris/bitstream/handle 
20. Brito KQD, Menezes TN, Olinda RA. Incapacidade funcional: condições de saúde e prática de atividade física em idosos. Rev Bras Enferm. 2016;69(5):825-32.

21. Azevedo PAC, Modesto CMS. A (re)organização do núcleo de cuidado familiar diante das repercussões da condição crônica por doença cardiovascular. Saúde Debate. 2016;40(110):183-94.

22. Sá PHVO, Cury GC, Ribeiro LCC. Atividade física de idosos e a promoção da saúde nas unidades básicas. Trab Educ Saúde. 2016;14(2):545-58.

23. Lee IM, Shiroma EJ, Lobelo F, Puska P, Blair SN, Katzmarzyk PT. Effect of physical inactivity on major noncommunicable diseases worldwide: an analysis of burden of disease and life expectancy. Lancet. 2012;380(9838):21929.

24. World Health Organization. A global brief on hypertension: silent killer, global public health crisis. Geneva: WHO; 2013 [acesso em 2018 Jun 30]. Disponível em: http://ish-world.com/downloads/pdf/global_brief_hypertension. pdf

25. Peckham S, Falconer J, Gillam S, Hann A, Kendall S, Nanchahal K, et al. The organisation and delivery of health improvement in general practice and primary care: a scoping study Health Serv Deliv Res [Internet]. 2015;3(29) [acesso em 2018 Jun 20]. Disponível em: https://www.ncbi.nlm.nih.gov/pubmed/26131542

26. Santos ROM. O vínculo longitudinal como dispositivo do cuidado: Saúde da Família e doenças crônicas em uma comunidade do Rio de Janeiro [dissertação]. Rio de Janeiro: Escola Nacional de Saúde Pública Sergio Arouca; 2015.

27. Brenk-Franz K, Strauss B, Tiesler F, Fleischhauer C, Ciechanowski P, Schneider N, et al. The influence of adult attachment on patient self-management in primary care - the need for a personalized approach and patientcentred care. PLoS One. 2015;10(9):e0136723.

28. Moraes SDTA, Buchalla CM, Valenti VE, Leite L, Tanaka ACA, Macedo H Júnior, et al. Acolhendo o acolhedor: o caminho mais curto para a humanização da assistência. Rev Bras Crescimento Desenvolv Hum [Internet] 2009 [acesso em 2018 Jun 13];19(3):393-402. Disponível: http://www.revistasusp.sibi.usp.br/pdf/ rbcdh/v19n3/06.pdf

29. Ministério da Saúde (BR). Política Nacional de Humanização - PNH. Brasília: Ministério da Saúde; 2013.

30. Lima CA, Oliveira APS, Macedo BF, Dias OV, Costa SM. Relação profissional-usuário de saúde da família: perspectiva da bioética contratualista. Rev Bioét. 2014;22(1):152-60.

31. Ministério da Saúde (BR), Secretaria de Atenção à Saúde, Departamento de Atenção Básica. Política Nacional de Atenção Básica. Brasília: Ministério da Saúde; 2017.

32. Muniz EA, Freitas CASL, Oliveira EN, Lacerda MR. Home care for the elderly in the family health strategy: perspectives on the care organization. Rev Enferm UFPE. 2017;11(Supl 1):296-302.

33. Ministério da Saúde (BR). Caderneta de saúde da pessoa idosa. Brasília: Ministério da Saúde; 2008 [acesso em 2018 Jun 20]. (Série A. Normas e Manuais Técnicos). Disponível em: http://bvsms.saude.gov.br/bvs/ publicacoes/ caderneta_saude_pessoa_idosa.pdf

34. Ministério da Saúde (BR), Secretaria de Atenção à Saúde, Departamento de Atenção Básica. Envelhecimento e saúde da pessoa idosa. Brasília: Ministério da Saúde; 2006. (Série A. Normas e Manuais Técnicos / Cadernos de Atenção Básica, n. 19).

\section{Endereço para correspondência:}

Andressa Christiny da Silva Ferreira

Universidade Federal de Mato Grosso - UFMT

Rodovia MT 130, Km 6

Bairro: Sagrada Família

CEP: 78005-050 - Rondonópolis - MT - Brasil

E-mail: andressa.christiny@hotmail.com 